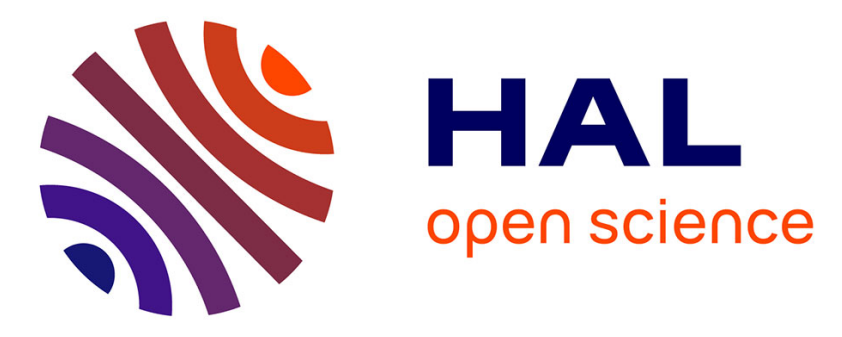

\title{
Mechanical Coupling in Gold Nanoparticles Supermolecules Revealed by Plasmon-Enhanced Ultralow Frequency Raman Spectroscopy
}

Adrien Girard, Hélène Gehan, Aurelien Crut, Alain Mermet, Lucien Saviot, Jeremie Margueritat

\section{To cite this version:}

Adrien Girard, Hélène Gehan, Aurelien Crut, Alain Mermet, Lucien Saviot, et al.. Mechanical Coupling in Gold Nanoparticles Supermolecules Revealed by Plasmon-Enhanced Ultralow Frequency Raman Spectroscopy. Nano Letters, 2016, 16 (6), pp.3843-3849. 10.1021/acs.nanolett.6b01314 . hal01320916

\section{HAL Id: hal-01320916 https://hal.science/hal-01320916}

Submitted on 31 May 2016

HAL is a multi-disciplinary open access archive for the deposit and dissemination of scientific research documents, whether they are published or not. The documents may come from teaching and research institutions in France or abroad, or from public or private research centers.
L'archive ouverte pluridisciplinaire HAL, est destinée au dépôt et à la diffusion de documents scientifiques de niveau recherche, publiés ou non, émanant des établissements d'enseignement et de recherche français ou étrangers, des laboratoires publics ou privés. 


\title{
Mechanical coupling in gold nanoparticles
}

\section{super-molecules revealed by}

\section{plasmon-enhanced ultra low frequency Raman}

\section{spectroscopy.}

\author{
A. Girard, ${ }^{\dagger}$ H. Gehan, ${ }^{\dagger}$ A. Crut, ${ }^{\dagger}$ A. Mermet, ${ }^{\dagger}$ L. Saviot, ${ }^{\ddagger}$ and J. Margueritat ${ }^{*} \dagger$ \\ †Institut Lumière Matière, Université de Lyon, Université Claude Bernard Lyon 1, UMR \\ CNRS 5306, 69622 Villeurbanne, France \\ $\ddagger$ Laboratoire Interdisciplinaire Carnot de Bourgogne, UMR 6303 CNRS-Université de \\ Bourgogne Franche Comté, 9 Av. A. Savary, BP 47 870, F-21078 Dijon Cedex, France \\ E-mail: jeremie.margueritat@univ-lyon1.fr
}

Phone: +33 (0)4 72448332

\begin{abstract}
Acoustic vibrations of assemblies of gold nanoparticles were investigated using ultra low frequency micro-Raman scattering and finite element simulations. When exciting the assemblies resonantly with the surface plasmon resonance of electromagnetically coupled nanoparticles Raman spectra present an ultra-low frequency band whose frequency lies below the lowest Raman active Lamb mode of single nanoparticles was observed. This feature was ascribed to a Raman vibration mode of gold nanoparticles "supermolecules", i.e. nanoparticles mechanically coupled by surrounding polymer molecules. Its measured frequency is inversely proportional to the nanoparticle diameter and sensitive to the elastic properties of the interstitial polymer. The latter
\end{abstract}


dependence as well as finite element simulations suggest that this mode corresponds to the out-of-phase semirigid translation ( $\ell=1$ Lamb mode) of each nanoparticle of a dimer inside the matrix, activated by the mechanical coupling between the nanoparticles. These observations were permitted only thanks to the resonant excitation with the coupling plasmon excitation, leading to an enhancement up to $10^{4}$ of the scattering by these vibrations. This enhanced ultra low frequency Raman scattering thus opens a new route to probe the local elastic properties of the surrounding medium.

\section{Keywords}

Vibration, Lamb, gold NPs dimer, mechanical properties, Raman, Brillouin

\section{Article}

During the last two decades many works have focused on the characterization of the acoustic vibrations of nanoparticles (NPs). Many materials were considered (dielectric spheres, ${ }^{1-4}$ metallic NPs,${ }^{5-7}$ quantum dots ${ }^{8-10}$ ) using different optical spectroscopy techniques such as

pump-probe experiment, ${ }^{11-14}$ and low frequency Raman or Brillouin scattering. ${ }^{7,15-18}$ They mostly addressed the localized acoustic vibration modes of non-interacting nano-objects, yielding many results regarding the effect of the NP crystallinity, ${ }^{7,15}$ size, ${ }^{16}$ shape, ${ }^{17,18}$ and surrounding medium. ${ }^{19,20}$

More recently, results on the physical properties of ordered NP assemblies were obtained. ${ }^{5,21-24}$ In analogy with their atomic counterparts, such assemblies are commonly called super-crystals (where atoms are replaced by spherical NPs) and also sustain long wavelength acoustic waves. These correspond to coherent motions of the NPs around their equilibrium positions, propagating with a velocity governed by the mass of the spheres and the elastic properties of the ligands that bind them together. Various pump-probe experiments ${ }^{25-29}$ have enabled the observation of such elastic waves, showing that they propagate at speeds lower 
than in their atomic crystal counterparts due to heavier masses and looser bonds. However, probing such sound waves in supercrystals through the classical Brillouin scattering technique has not yet been reported, most probably due to their small scattering cross-sections. Yet since more than 40 years, the limitation due to poor scattering efficiencies in light scattering experiments is known to be possibly lifted by placing the object of interest in the close vicinity of a metallic surface, like in surface enhanced Raman scattering. ${ }^{30}$ Such a possibility was recently demonstrated for low frequency vibration modes of NPs. ${ }^{31,32}$

In the present paper the possibility to enhance the scattering by ultra low frequency modes was investigated, using gold NPs where collective electron oscillations (localized plasmons) and collective atom oscillations (localized vibrations) take place at the same time within the same object, which thus acts both as enhancer and scatterer. In particular, using an excitation wavelength corresponding to the optical resonance of gold NPs in close vicinity enables to measure new low frequency features that are characteristic of the mechanical coupling between the NPs.

The solutions of gold NPs used in this study were synthesized following a seeded-growth procedure already reported in the literature ${ }^{33,34}$ (see Supporting Information for a detailed description). Thanks to this method it was possible to tune the gold NPs diameters from few to hundred of nanometers. After synthesis, polyvinylpyrrolidone (PVP) was used to stabilize the as-synthesized gold NPs in order to transfer them in ethanol, ensuring colloidal stability over several months. For optical measurements, drops of gold NP/PVP solutions were deposited onto indium tin oxide substrates and let dry overnight, leading to the formation of a polymer film with a thickness of approximately $100 \mu \mathrm{m}$ in which the NPs are embedded. Meanwhile, drops of the same solutions diluted one hundred times in ethanol were deposited onto carbon grids for morphological characterization using a transmission electron microscope (TEM). The average NP diameter in each solution was found to vary from 12 to $100 \mathrm{~nm}$, with relatively low size dispersion (see Supporting Information for a detailed description).Typical images of gold NPs from a solution with average diameters of 
$100 \mathrm{~nm}$ are presented in Figure 1 A and B. For this sample the size dispersion is about 10\%. The entanglement of adsorbed PVP chains of neighboring NPs during the drying step leads to the formation of disordered clusters, with a 1 to $2 \mathrm{~nm}$ inter-particle separation due to the polymer coating that prevents a direct contact between the particles. As an example the dimer of Figure 1B shows an inter-particle distance $(d)$ of about $1 \mathrm{~nm}$.

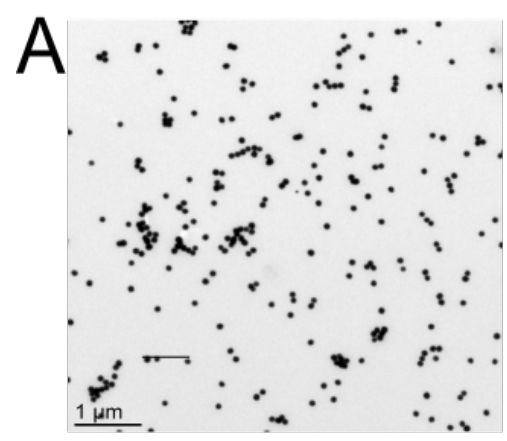

C

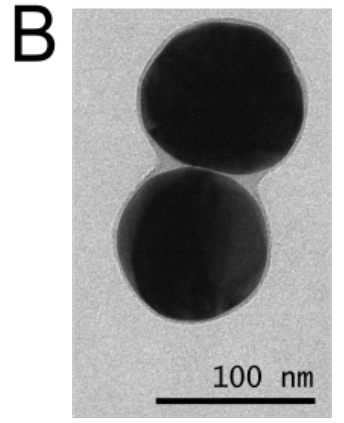

Absorption

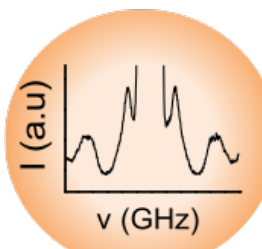

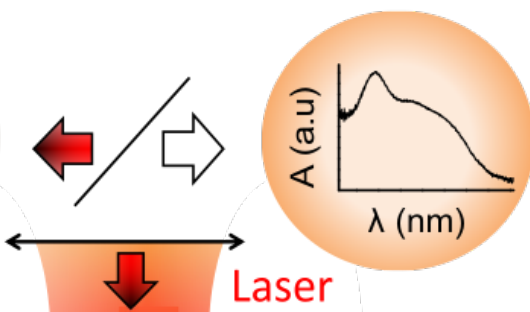

PVP $80 \% 8$ AunPs $\uparrow 100 \mu \mathrm{m}$

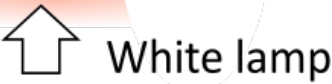

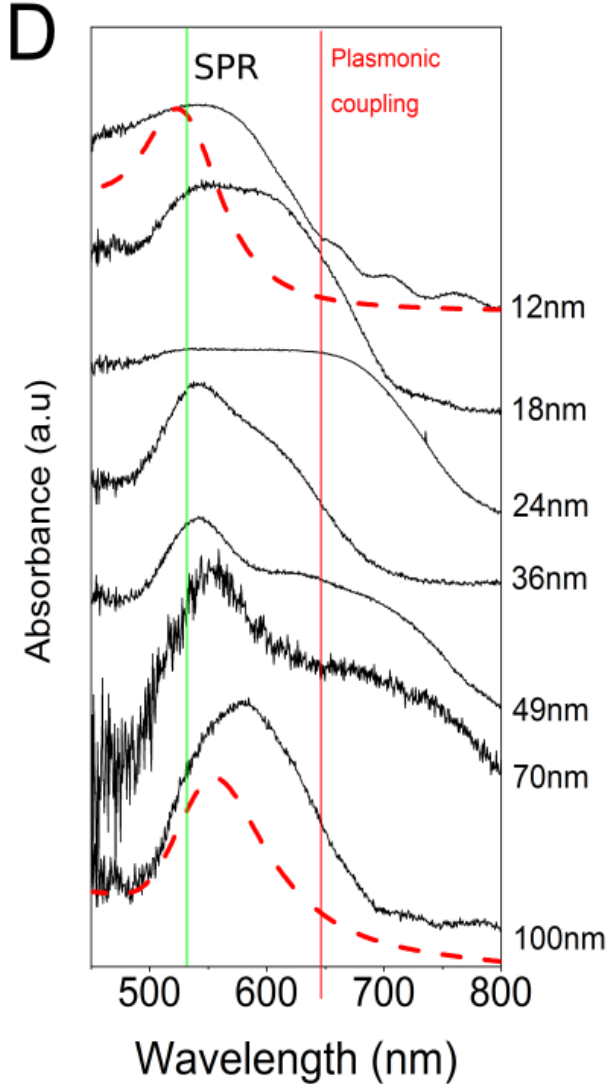

Figure 1: A. TEM image of diluted gold $100 \mathrm{~nm}$ sample. B. $100 \mathrm{~nm}$ NP dimer showing $d \approx 0.8 \mathrm{~nm}$ inter-particle spacing. C. Experimental setup allowing to measure both micro-absorption (transmission geometry, white lamp) and micro-Raman spectra (backscattering geometry, 532/647 nm laser lines) of gold NPs from a unique sample spot (laser spot $\left.\approx 0.6 \mu \mathrm{m}^{2}\right)$. D. From top to bottom, absorption spectra of samples with 12 to $100 \mathrm{~nm}$ diameter showing the dipolar resonance of isolated particles and the plasmonic coupling band. Red dashed lines correspond to absorption spectra of 12 and $100 \mathrm{~nm}$ NPs in liquids before deposition. Green and red vertical lines correspond to the 532 and $647 \mathrm{~nm}$ excitation wavelengths used for Raman spectroscopy, respectively.

Micro-absorption and micro-Raman spectra were measured at the same position as sketched 
in Figure 1C, allowing to correlate plasmonic and vibrational resonances of the assemblies. Absorption spectra were recorded in transmission configuration. The white light beam was focused onto the sample surface using a condenser with a numerical aperture of 0.65 and the transmitted light was collected with a $\times 100$ objective $(\mathrm{NA}=0.95)$ and focused onto the entrance slit of a spectrometer equipped with a thermo-electric cooled CCD.

Figure 1 D shows the absorption spectra obtained for seven films of PVP containing NPs with different average diameters, together with those of the initial solutions of PVPcoated NPs for $12 \mathrm{~nm}$ and $100 \mathrm{~nm}$ diameters. A typical band around $540 \mathrm{~nm}$ is observed in all spectra and is attributed to the dipolar surface plasmon resonance of isolated NPs, which is confirmed by its presence on the spectra obtained in liquids. At larger wavelengths, between 600 and $750 \mathrm{~nm}$, one additionally observes a more or less well defined shoulder that is not observed in the initial solution (Figure 1 D)(see also Supporting Information). The origin of this additional feature lies in the large density of NPs and the presence of NPs clusters with short interspacing distance $(d)$ (Figure 1C), leading to a strong electromagnetic coupling between neighbouring NPs and thus the appearance of additional resonances in the optical response of the assemblies ${ }^{35-38}$ (referred to in the following as "coupling plasmon band") . In the simplest case of NP dimers, it was shown that the energy of the coupling plasmon band redshifts as the gap size decreases. ${ }^{39}$ For NP assemblies, the energy of the coupling plasmon band and its relative intensity depend on different parameters such like the distribution of interparticle spacing, the ratio of interacting versus non interacting NPs and the size of the NPs. These multiple dependences explain why no clear trend in the intensity of the plasmonic coupling band is observed as the diameters of the NPs increase in Figure 1 D. Nevertheless the absorbance is always non-negligible around $647 \mathrm{~nm}$ implying that significant electromagnetic interaction between NPs take place in all samples. This is in agreement with Figure 1 B which underlines the possibility to form supermolecules, among which the basic one is the dimer, with an average interparticle separation of about $1 \mathrm{~nm}$ fixed by the PVP coating shell. In the following, we focus on how tuning the excitation wavelength 
to the plasmonic coupling band of these NP assemblies allows to detect the inelastic light scattering from interacting NPs vibration modes.

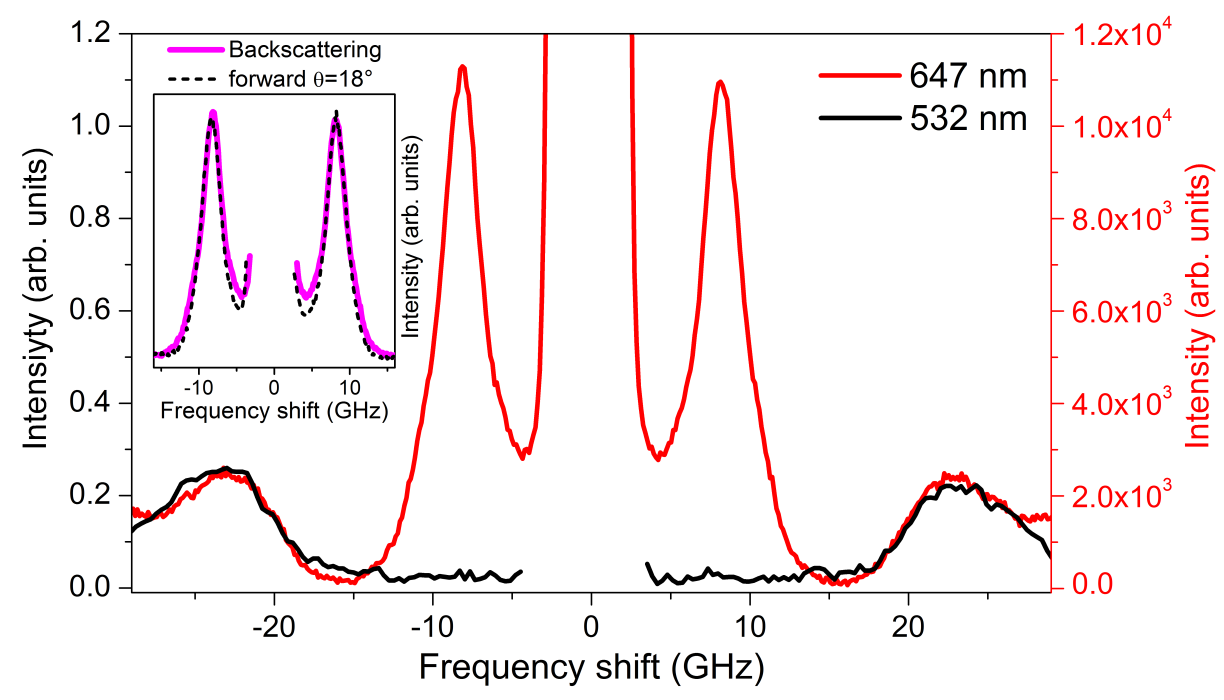

Figure 2: Raman spectra of the Au49nm sample recorded at $532 \mathrm{~nm}$ (resonance with the surface plasmon resonance (black curve and scale)) and at $647 \mathrm{~nm}$ (resonance with the plasmon coupling band (red curve and scale)). The spectra were normalized with respect to the acquisition duration and power. The inset shows spectra of the same sample recorded in backscattering geometry $\left(\theta_{\vec{k}_{i n c}, \vec{k}_{s c a}}=180^{\circ}\right)$ (pink full line) and forward scattering geometry at $\theta_{\vec{k}_{i n c}, \vec{k}_{s c a}}=18^{\circ}$ (black dashed line).

Low frequency Raman scattered light was collected in the backscattering geometry using a $\times 100$ objective $(\mathrm{NA}=0.95)$ and focused on the entrance hole of a six pass tandem FabryPérot interferometer that can be operated at two distinct wavelengths. These wavelengths were chosen to match either the dipolar resonance of the isolated NPs or the plasmonic coupling band of the interacting NPs and generated using the $532 \mathrm{~nm}$ line from a solid state laser and the $647 \mathrm{~nm}$ line from a krypton laser (vertical green and red lines in Figure 1D). Figure 2 shows the Raman spectra of a sample containing gold NPs of $49 \mathrm{~nm}$ diameter for both excitations. At $532 \mathrm{~nm}$ the Raman spectrum shows no other contribution than the quadrupolar $\ell=2 \mathrm{Lamb}$ mode at $22 \mathrm{GHz}$, in excellent agreement with its expected 
frequency for free nanospheres $f(\ell=2)=\frac{0.85 v_{T}}{D}=21.6 \mathrm{GHz}$ using the transverse velocity of bulk gold $v_{T}=1250 \mathrm{~m} \cdot \mathrm{s}^{-1}$ and the average diameter $(D)$ measured by TEM. When tuning the laser wavelength to $647 \mathrm{~nm}$ close to the resonance with the plasmonic coupling band, the quadrupolar vibration mode is still observed at the same frequency. However, the spectra of Figure 2, normalized with respect to the acquisition time and laser power used, show that the measured intensity of the $\ell=2$ mode is at least 1000 times larger with the red excitation than with the green excitation, evidencing the strong enhancement in the former case (see Supporting Information). The strong enhancement observed is probably due to the higher sensitivity of the absorption cross-section to the small displacement of the nanoparticles surface at $647 \mathrm{~nm}$.

Additionally, the resonant excitation with the coupling plasmon band also leads to the observation of a second sharp and intense band in the low frequency part of the spectra around $9 \mathrm{GHz}$. Its frequency stands largely below that of the traditionally observed lowest frequency Raman active mode, ${ }^{40}$ i.e. the quadrupolar $\ell=2$ mode. This ultra low frequency range is reminiscent of previous pump-probe spectroscopy measurements on super-crystals in which a mode correlated to the propagation of acoustic waves between the mechanically interacting NPs was observed. ${ }^{25,27,28}$ However the independence of the ultra low frequency peak with scattering angle (Figure 2 inset) confirms the confined (rather than propagative) character of the vibration mode observed.

The ultra low Raman spectra obtained with varying NP diameters from 12 to $100 \mathrm{~nm}$ are presented in Figure 3A. For all spectra the typical acquisition times were of the order of one hour except for the sample containing NPs of $12 \mathrm{~nm}$ diameter (24 hours), which yielded a low scattering efficiency, as illustrated by the lower signal-to-noise ratio and the emergence of a $26 \mathrm{GHz}$ Brillouin signal from the underneath substrate. Note that the usually intense Brillouin signal is completely screened by the intense ultra low frequency band otherwise. Both the quadrupolar and the ultra low frequency band frequencies decrease with increasing NP diameters(Figure 3B). The dependence of the quadrupolar frequency measured with 

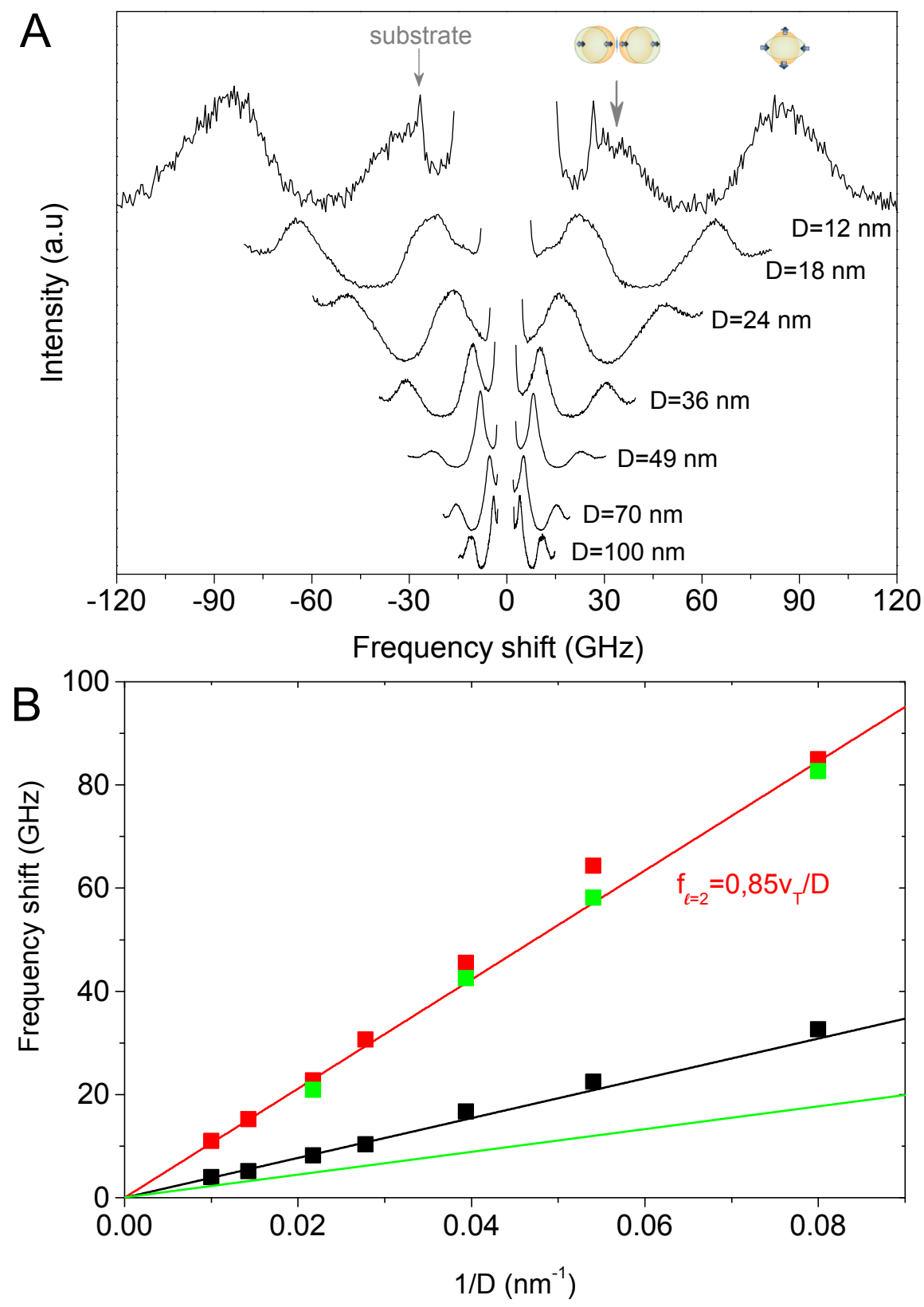

Figure 3: A. Low and ultra low frequency Raman spectra of NPs assemblies with varying NPs diameters $(12-100 \mathrm{~nm})$. B. Frequency shift as a function of the NP inverse diameter for the ultra low frequency band at $647 \mathrm{~nm}$ (black squares), the quadrupolar $\ell=2$ vibration bands at $532 \mathrm{~nm}$ (green squares) and $647 \mathrm{~nm}$ (red squares). Red full line is the theoretical quadrupolar frequency $f=0.85 \mathrm{v}_{T} / \mathrm{D}$ and the black line is a fit of the ultra low frequency band with zero intercept. $\ell=1$ fundamental frequency, calculated with complex frequency model ${ }^{6}$ in PVP matrix, is indicated with a green line. 
the $532 \mathrm{~nm}$ excitation wavelength, i.e. exciting the dipolar resonance, scales as $0.85 v_{T} / D$ as predicted by the free sphere Lamb model. ${ }^{41}$ With the $647 \mathrm{~nm}$ excitation the frequency variation slightly deviates from the Lamb model prediction, in particular for the NPs of 25 and $18 \mathrm{~nm}$ diameters. These deviations will be explained thereafter at the light of the interpretation of the ultra low frequency band.

The ultra low frequency band position also scales inversely to the NP diameter, but with a lower slope as compared to the $\ell=2$ mode. This means that this vibration mode is somehow related to the vibration of the NPs. Only spheroidal and torsional $\ell=1$ Lamb modes can reach such a low frequency. For a free sphere the frequencies of these modes are zero since there is no restoring force. They become non-zero for NPs embedded in a host matrix, and correspond to rattling (constrained translation) and libration (constrained rotation) vibrations respectively. This latter mode is never observed in Raman due to its torsional nature and is discarded in the following. According to selection rules ${ }^{40}$ the rattling mode of a single NP is not Raman active. However it is instructing to compare the frequency of the ultra low frequency mode with the rattling mode of a single NP since it is well known that in the case of strong enhancement with hot-spots, Raman selection rules may be lifted. To do so we used the complex frequency model described elsewhere ${ }^{6}$ considering a gold particle in an infinite PVP matrix. The transverse and longitudinal velocities as well as the density of bulk gold were used. The density of PVP used for this calculation was $\rho=1.2 \mathrm{~g} . \mathrm{cm}^{-3}$ and its longitudinal and transverse sound velocities $\left(\mathrm{v}_{L}=2989 \mathrm{~m} \cdot \mathrm{s}^{-1}\right.$ and $\left.\mathrm{v}_{T}=1443 \mathrm{~m} . \mathrm{s}^{-1}\right)$ were deduced from Brillouin experiments on gold-free PVP films (see Supporting Information). The calculated spheroidal $(\ell=1, n=0)$ frequencies are reported in Figure $3 \mathbf{B}$ (green line). However, these calculated frequencies do not reproduce the experimental frequencies. The ultra low frequency mode observed can therefore not be simply assigned to the spheroidal $\ell=1$ eigenmode of an isolated NP even if it appears close enough to the experimental values.

Another possibility for the rattling mode to become Raman active is a change of the symmetry of the system. This occurs for example when placing a NP on a surface ${ }^{42}$ or, when 
considering a supermolecule like a dimer of $\mathrm{NPs}^{43}$ in which the frequency of the vibration will be necessarily impacted. Two dimer modes can be built from the rattling modes of the individual embedded NPs, namely the in-phase and the out-of-phase rattling motions of the NPs. From symmetry considerations, only the out-of-phase mode is expected to be Raman active. Since the separation between the two NPs oscillates in the vibration, we expect a strong coupling of this mode with the coupling plasmon band. The ultra low frequency feature could thus be the signature of the mechanical coupling between both NPs (mediated by the PVP matrix) resulting in dimer supermolecule modes.

This ultra low frequency vibration should therefore depend on the elasticity of the interstitial polymer (PVP). In order to check this assumption we have followed the evolution of the low frequency Raman spectra as a function of temperature. We have measured the Raman spectra of the sample containing gold NPs of $49 \mathrm{~nm}$ diameter from ambient temperature $\mathrm{T}=24^{\circ} \mathrm{C}$ down to $\mathrm{T}=-180^{\circ} \mathrm{C}$. In this temperature range, the $\mathrm{PVP}$ matrix remains in the vitreous state but its elastic properties are modified. The corresponding Raman spectra are gathered in Figure 4A while Figure 4B compares the relative frequency shifts of the Raman bands with those of a NP-free PVP bulk film drop-cast in the same conditions as the NP containing samples. Upon cooling all vibration frequencies increase. For a free sphere, the expected frequency shift of the quadrupolar $\ell=2$ mode results from the variation of the density $\rho$ and from the elastic constant $\mathrm{C}_{44}$ of gold, which accounts for the relative variation: $\frac{\Delta f}{f}=\frac{1}{2}\left(\frac{\Delta C_{44}}{C_{44}}-\frac{\Delta \rho}{\rho}\right)$. The coefficient of thermal expansion of gold is $\alpha_{V}^{\text {gold }}=1.4 \times 10^{-5} \mathrm{~K}^{-1}$, which results in a mass density variation of only $\frac{\Delta \rho}{\rho}=\alpha_{V}^{\text {gold }} \Delta T=0.3 \%$ over the considered temperature range. From Ref. 44, the relative variation of $C_{44}$ is about $5.7 \%$ in the 0 -300 K range. This leads to $\frac{\Delta f}{f} \approx 2.7 \%$, in very good agreement with the measured variation $\frac{\Delta f_{\ell=2}}{f_{\ell=2}}=2.8 \%$. Therefore the moderate frequency shift of the $\ell=2$ mode is mostly related to the temperature-induced gold stiffening, in agreement with calculations using the complex frequency model which also show that its frequency is weakly affected by the elastic properties of the environnement. 
A
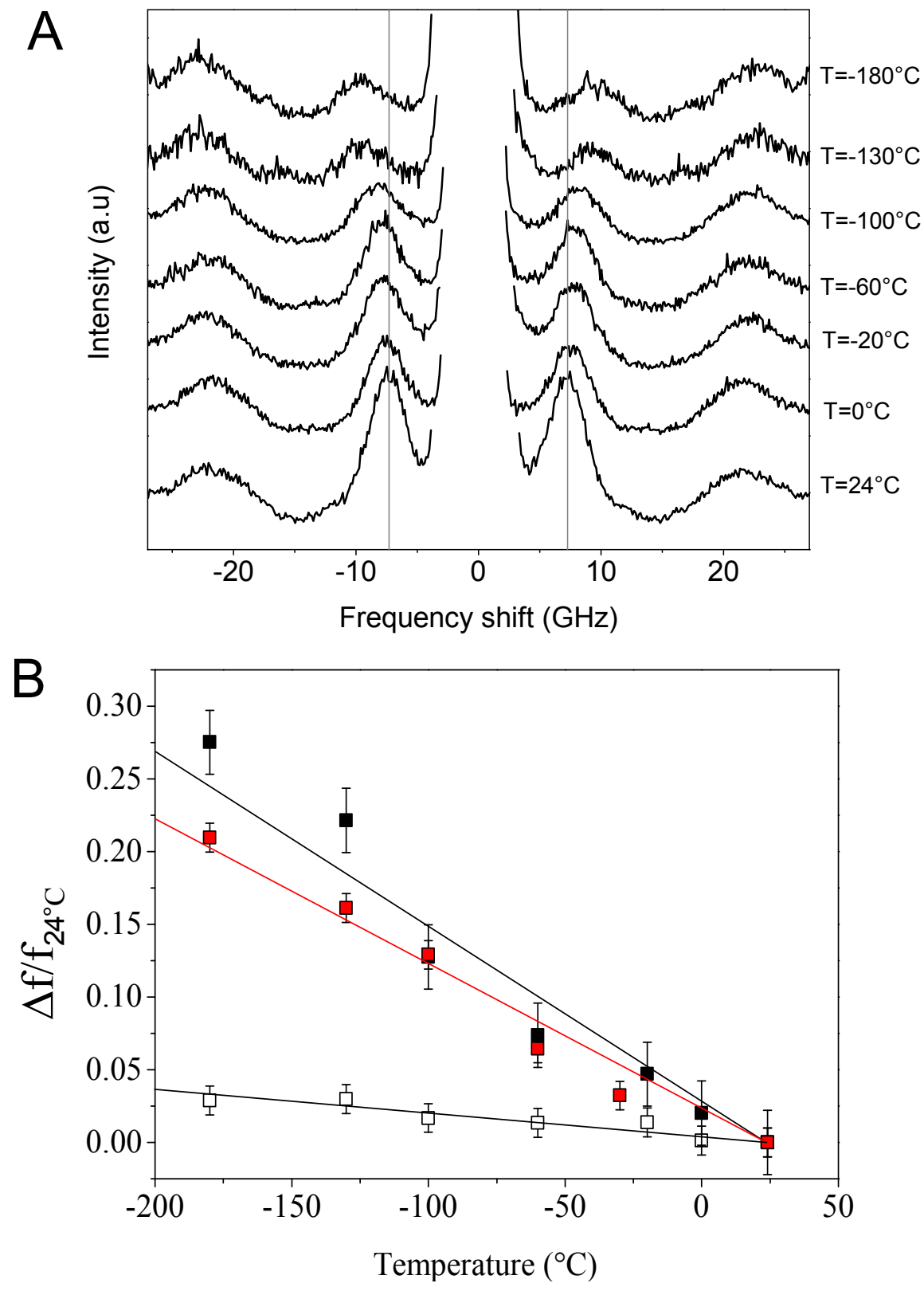

Figure 4: A. Au49nm Raman spectra upon decreasing temperature from $24^{\circ} \mathrm{C}$ down to $-180^{\circ} \mathrm{C}$ from bottom to top. B. Relative frequency shifts normalized to those of ambient temperature for the $\ell=2$ (empty squares), the ultra low frequency mode (black squares) and the PVP longitudinal Brillouin mode (red square). 
Conversely, the ultra low frequency band (black squares) strongly deviates from this trend with a relative shift $\frac{\Delta f}{f}=27.5 \%$. This further suggests that this mode cannot be interpreted as a Lamb mode of a single NP. In order to evaluate the polymer contribution, the longitudinal Brillouin mode of the PVP without NPs was monitored and is reported in Figure 4B(red squares). Over this temperature range, a relative increase of the Brillouin longitudinal mode frequency by $21 \%$ is observed. Adding the experimental frequency variation due to the gold elastic parameters $(21+2.8 \approx 24 \%)$ results in a value close to the measured one. Therefore, the ultra low frequency mode is strongly related to the PVP which acts as a binder between NPs.

Since we have strong evidence that the lowest frequency vibrations observed in the Raman spectra are due to the coupling between NPs, we consider in the following the case of dimer vibrations, which is the simplest way to take into account interparticle coupling between adjacent NPs in supermolecules. This does not necessarily imply that the measured Raman intensity comes from dimers only.

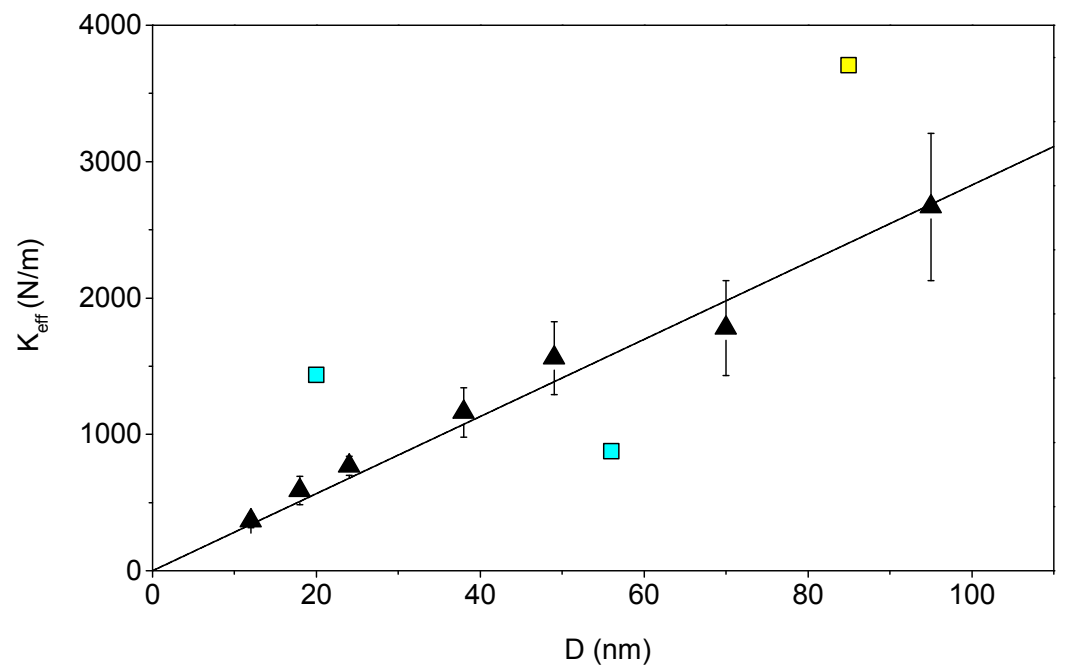

Figure 5: Size dependence of the effective stiffness constant $K_{\text {eff }}$ evaluated from the spring model (black triangles). Cyan and yellow squares are data from ref. ${ }^{45}$ and ref. ${ }^{46}$

The vibration of interest can be modeled in a first approach by the symmetric stretch of two masses connected by a spring of effective stiffness $K_{\text {eff }}$. Within this simple picture the 
dimer vibrationnal frequency can be approximated by :

$$
\omega=\sqrt{\frac{2 K_{e f f}}{M}}
$$

where $\mathrm{M}=\rho \frac{4}{3} \pi\left(\frac{D_{N P}}{2}\right)^{3}$ is the mass of one NP. The effective stiffness constants $K_{\text {eff }}$ evaluated from equation 1, ranging between $370 \mathrm{~N} . \mathrm{m}^{-1}$ and $2700 \mathrm{~N} . \mathrm{m}^{-1}$, as a function of the NPs diameters are summarized in Figure 5 and compared to previous measurements performed using pump-probe spectroscopy on isolated dimers containing $80 \mathrm{~nm},{ }^{46} 28$ and $10 \mathrm{~nm}$ diameter gold particles. ${ }^{45}$ The values of $K_{\text {eff }}$ deduced from these measurements are of the same order of magnitude as ours (Figure 5), even if in these references the authors claimed a direct contact between the gold NPs. Moreover the observed linear behavior is in good agreement with studies conducted on crystals of coupled large polystyrene spheres in contact. ${ }^{1}$ However, the spring model cannot be expected to provide a good description of the observed low frequency mode as the non-zero frequency of the NP rattling mode was shown to be a consequence of the full embedding of the NPs. ${ }^{43}$ Moreover, this model only provides a rough approximation of the dimer nano-contact as it does not allow taking into account subtle effects such as the elastic stiffening of the surrounding medium due to the interaction with the NP surface. ${ }^{47-50}$ More importantly, the spring model assumes a purely stretching motion of rigid spheres, while the actual rattling motion involves a dipolar-type deformation of the nanospheres.

In this work the entanglement of the PVP molecules form a solid vitreous matrix all around the sphere. In order to fully take into account the presence of such an embedding matrix we have used finite element modeling (Figure $6 \mathbf{A}$ ). In this approach, a gold dimer is set within a spherical embedding medium of larger dimensions with the elastic properties of PVP. A spherical perfectly matched layer prevents any unrealistic effect due to the reflection of acoustic waves at the system boundaries. An oscillating unidirectional boundary load is applied on one NP side in the direction of the dimer long axis and varying the frequency of 

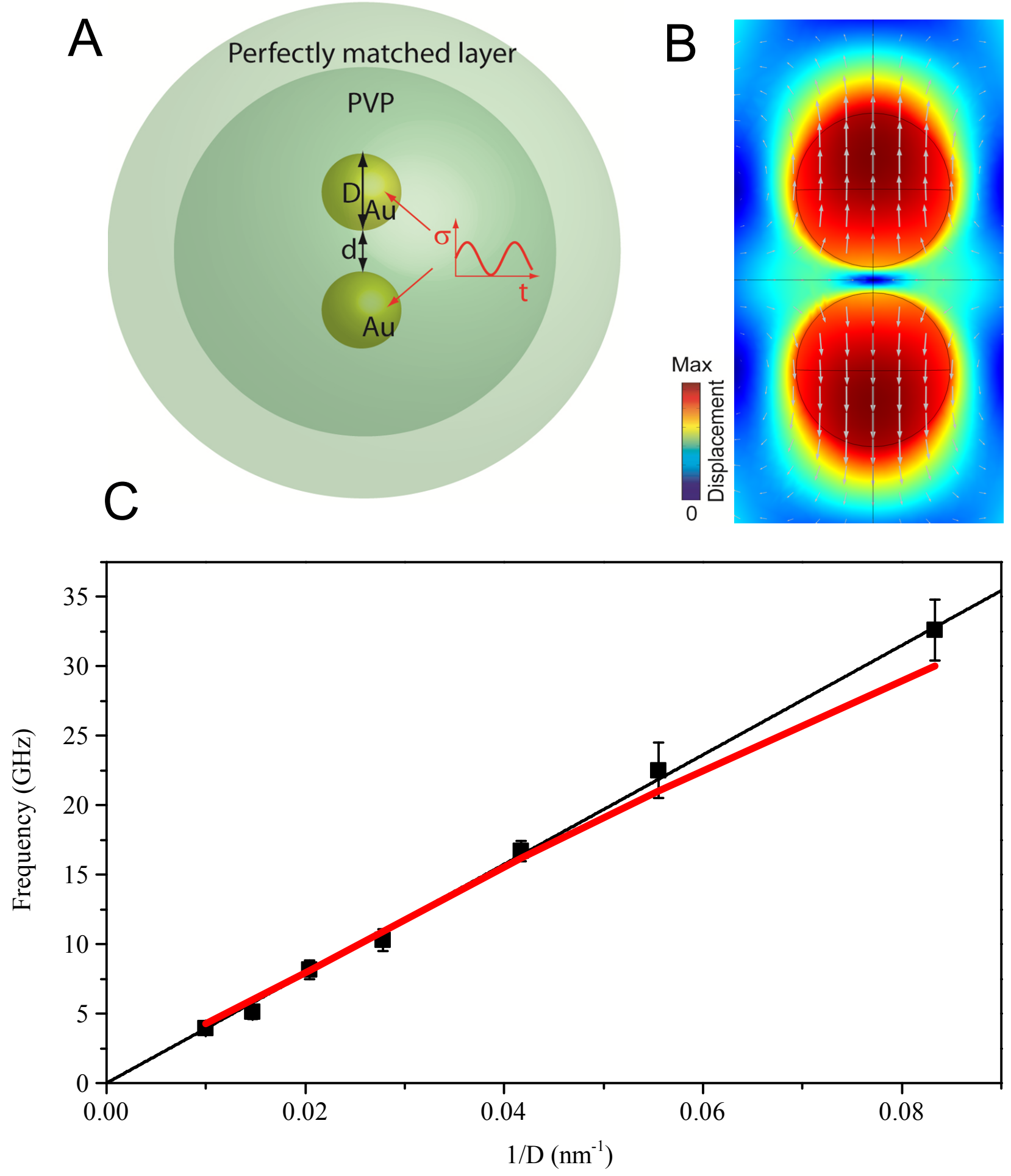

Figure 6: A. Schematic view of the simulation geometry. B. Representation of the dimer out of phase vibration mode. C. Calculated dimer out of phase (red) frequencies as a function of the inverse NP diameter compared to experimental frequencies (black squares). 
this excitation allows to probe the different acoustic resonances of the system, considering the energy stored in the gold NPs or the displacement amplitude (See Supporting Information for more details).

The calculations were performed using the same parameters as those used for estimating the rattling mode frequencies of single NPs (Figure 3). As an illustration the dimer eigenmode (relevant for this study) is sketched Figure 6B. for a $12 \mathrm{~nm}$ diameter NP. This mode corresponds to an out-of-phase translation of the NP centers, i.e. a symmetric stretch of the dimer, with a slight dipolar deformation of the NPs. They are mainly translated along the dimer symmetry axis, which confirms their link with the spheroidal $\ell=1$ mode of a single sphere in a matrix. As shown in Figure $6 \mathbf{C}$. the calculated size dependence of the out of phase mode frequency is found to perfectly match the experimental one. Note that the excellent agreement between measured and computed vibrational frequencies is not preserved if simulations are performed on more complex multimers (e.g. trimers), a posteriori justifying the assumption of dimeric acoustic interactions made in the modeling. In order to appreciate the effect of the different parameters used in the model, a typical variation of $10 \%$ around the values used here $\left(d=1 \mathrm{~nm}, \mathrm{C}_{11}^{P V P}=10.7 \mathrm{GPa}, \rho_{P V P}=1200 \mathrm{~g} / \mathrm{m}^{3}\right.$, and $\left.\nu_{P V P}=0.35\right)$ was applied to each of them. These calculations (detailed in the Supporting Information) show that $d, \rho_{P V P}$, and $\nu_{P V P}$ only have a weak impact on the relative frequency shift of the dimer vibration mode (less than $1 \%$ ) compared to the $\mathrm{C}_{11}^{P V P}$ that induces a variation of $5 \%$. These results confirm that the dimer vibration mode that originates from the mechanical coupling of the $\ell=1$ rattling mode is essentially sensitive to the elastic properties of the surrounding medium. Basically what we have observed with the rattling mode at ultra low frequencies is a classical coupling scheme where two identical modes of moieties couple to generate an out-of-phase mode and a (herein non-observed) in-phase mode, the frequency of the out-of-phase mode being larger than that of the non-coupled moieties. In the present experiment with gold NPs the effect of this coupling was observed thanks to the resonance between the Raman excitation wavelength and the plasmonic coupling excitation. Therefore 
when the $\ell=2$ mode is detected with the $647 \mathrm{~nm}$ line, i.e. in resonance with the plasmon coupling band, the scattered signal is mainly due to the NPs that are present in resonant structures, i.e. mechanically coupled NPs thus explaining the small deviations observed for the $\ell=2$ mode frequencies in Figure 3 B. For the smallest NPs almost no coupling plasmon band is observed (Figure 1D) which means that there is only few coupled NPs and weak enhancement is observed, thus the signal measured is mainly due to the mechanically isolated NPs like in the case of an excitation at $532 \mathrm{~nm}$. Once again, thanks to the plasmonic enhancement it is thus possible to demonstrate the effect of the mechanical coupling on the vibration modes of mechanically coupled NPs.

In summary, we have successfully investigated the vibrations of polymer-connected gold NP supermolecules using ultra low frequency Raman spectroscopy, optical absorption measurements and finite element simulations. Tuning the excitation wavelength to the plasmonic coupling band has led to a great enhancement of Raman signals and allowed us to observe for the first time the inelastic scattering by an ultra low frequency vibrational mode of embedded and mechanically coupled gold NP supermolecules. Using finite element simulations, this vibration mode was assigned to an out of phase symmetric stretch of the NPs composing a dimer. We have demonstrated that this mode takes its origin in the spheroidal $\ell=1$ vibration mode (rattling) of embedded isolated NPs. The plasmon-enhanced spectroscopy of the vibrations of mechanically coupled NPs allows studying the elastic properties of the surrounding medium, as the dimer vibration mode was found to be sensitive to its change of elastic properties induced by temperature. The strong enhancement observed by exciting in resonance with the plasmonic coupling band opens the door to studying the vibrations of a single dimer and correlating its Raman spectrum with its exact morphology.

\section{Acknowledgement}

This work was supported by the ANR NanoVip project, grant ANR.13.JSIO.0002 of the 
French Agence National de la Recherche and the Fédération André Marie Ampère 2013

(FRAMA). The authors declare no competing financial interest. We thank the Centre Technologique des Microstructures at Villeurbanne $(\mathrm{CT} \mu)$ for access to the microscopes platform for TEM characterizations. We also thank E. Cottancin for his help for the morphological characterization by TEM.

\section{Supporting Information Available}

Details of experimental procedures and characterization data for the samples measured as well as details for finite element simulations can be found here.

This material is available free of charge via the Internet at http://pubs.acs.org/.

\section{References}

(1) Mattarelli, M.; Montagna, M.; Still, T.; Schneider, D.; Fytas, G. Soft Matter 2012, 8, 4235 .

(2) Li, Y.; Lim, H. S.; Ng, S. C.; Wang, Z. K.; Kuok, M. H.; Vekris, E.; Kitaev, V.; Peiris, F. C.; Ozin, G. Appl. Phys. Lett. 2006, 88, 1-3.

(3) Kuok, M. H.; Lim, H. S.; Ng, S. C.; Liu, N. N.; Wang, Z. K. Phys. Rev. Lett. 2003, 90, 255502.

(4) Palpant, B.; Portales, H.; Saviot, L.; Lermé, J.; Prével, B.; Pellarin, M.; Duval, E.; Perez, A.; Broyer, M. Phys. Rev. B 1999, 60, 17107-17111.

(5) Courty, A.; Mermet, A.; Albouy, P.; Duval, E.; Pileni, M. P. Nat. Mater. 2005, 4, 395-8.

(6) Murray, D. B.; Saviot, L. Phys. Rev. B 2004, 69, 094305. 
(7) Portales, H.; Goubet, N.; Saviot, L.; Adichtchev, S.; Murray, D. B.; Mermet, A.; Duval, E.; Pileni, M.-P. Proc. Natl. Acad. Sci. U. S. A. 2008, 105, 14784-9.

(8) Saviot, L.; Champagnon, B.; Duval, E.; Ekimov, A. Phys. Rev. B 1998, 57, 341-346.

(9) Saviot, L.; Champagnon, B.; Duval, E.; Kudriavtsev, I.; Ekimov, A. J. Non. Cryst. Solids 1996, 197, 238-246.

(10) Ivanda, M.; Babocsi, K.; Dem, C.; Schmitt, M.; Montagna, M.; Kiefer, W. Phys. Rev. B 2003, 67, 235329.

(11) Cardinal, M. F.; Mongin, D.; Crut, A.; Maioli, P.; Rodríguez-González, B.; PérezJuste, J.; Liz-Marzán, L. M.; Del Fatti, N.; Vallée, F. J. Phys. Chem. Lett. 2012, 3, 613-619, PMID: 26286157.

(12) Baida, H.; Christofilos, D.; Maioli, P.; Crut, A.; Del Fatti, N.; Vallée, F. J. Raman Spectrosc. 2011, 42, 1891-1896.

(13) Crut, A.; Maioli, P.; Del Fatti, N.; Vallée, F. Ultrasonics 2015, 56, 98-108.

(14) Crut, A.; Juvé, V.; Mongin, D.; Maioli, P.; Del Fatti, N.; Vallée, F. Phys. Rev. B 2011, $83,205430$.

(15) Portales, H.; Goubet, N.; Sirotkin, S.; Duval, E. Nano Lett. 2012, 12, 5292-5298.

(16) Bachelier, G.; Margueritat, J.; Mlayah, A.; Gonzalo, J.; Afonso, C. Phys. Rev. B 2007, $76,1-6$.

(17) Margueritat, J.; Gonzalo, J.; Afonso, C. N.; Mlayah, A.; Murray, D. B.; Saviot, L. Nano Letters 2006, 6, 2037-2042.

(18) Large, N.; Saviot, L.; Margueritat, J.; Gonzalo, J.; Afonso, C. N.; Arbouet, A.; Langot, P.; Mlayah, A.; Aizpurua, J. Nano Lett. 2009, 9, 3732-8. 
(19) Voisin, C.; Christofilos, D.; Del Fatti, N.; Vallée, F. Physica B Condens Matter 2002, 316-317, 89-94.

(20) Crut, A.; Maioli, P.; Del Fatti, N.; Vallée, F. Phys. Rep. 2015, 549, 1-43.

(21) Taleb, A.; Petit, C.; Pileni, M. P. J. Phys. Chem B 1998, 102, 2214-2220.

(22) Petit, C.; Taleb, A.; Pileni, M.-P. Chem. Eng. Technol. 1998, 21, 679-681.

(23) Pileni, M. P. Journal of Physics D: Applied Physics 2008, 41, 134002.

(24) Taleb, A.; Silly, F.; Gusev, A. O.; Charra, F.; Pileni, M. P. Advanced Materials 2000, 12, 633-637.

(25) Ruello, P.; Ayouch, A.; Vaudel, G.; Pezeril, T.; Delorme, N.; Sato, S.; Kimura, K.; Gusev, V. E. Phys. Rev. B 2015, 92, 174304.

(26) Huang, W.; Qian, W.; El-Sayed, M. A. Nano Lett. 2004, 4, 1741-1747.

(27) Lisiecki, I.; Polli, D.; Yan, C.; Soavi, G.; Duval, E.; Cerullo, G.; Pileni, M.-P. Nano Lett. 2013, 13, 4914-9.

(28) Lisiecki, I.; Halté, V.; Petit, C.; Pileni, M.-P.; Bigot, J.-Y. Adv. Mater. 2008, 41764179 .

(29) Mante, P.-A.; Chen, H.-Y.; Lin, M.-H.; Wen, Y.-C.; Gwo, S.; Sun, C.-K. Appl. Phys. Lett. 2012, 101, 101903.

(30) Margueritat, J.; Gehan, H.; Grand, J.; Lévi, G.; Aubard, J.; Félidj, N.; Bouhelier, A.; Colas-Des-Francs, G.; Markey, L.; Marco De Lucas, C.; Dereux, A.; Finot, E. ACS nano 2011, 5, 1630-8.

(31) Margueritat, J.; Gonzalo, J.; Afonso, C. N.; Hörmann, U.; Van Tendeloo, G.; Mlayah, A.; Murray, D. B.; Saviot, L.; Zhou, Y.; Hong, M. H.; Luk'yanchuk, B. S. Nanotechnology 2008, 19, 375701. 
(32) Tripathy, S.; Marty, R.; Lin, V. K.; Teo, S. L.; Ye, E.; Arbouet, A.; Saviot, L.; Girard, C.; Han, M. Y.; Mlayah, A. Nano Lett. 2011, 431-437.

(33) Ziegler, C.; Eychmüller, A. J. Phys. Chem. C 2011, 115, 4502-4506.

(34) Turkevich, J.; Stevenson, P. C.; Hillier, J. Discuss. Faraday Soc. 1951, 11, 55-75.

(35) Marhaba, S.; Bachelier, G.; Bonnet, C.; Broyer, M.; Cottancin, E.; Grillet, N.; Lermé, J.; Vialle, J.-L.; Pellarin, M. J. Phys. Chem. C 2009, 113, 4349-4356.

(36) Cha, H.; Yoon, J. H.; Yoon, S. ACS Nano 2014, 8, 8554-63.

(37) Khlebtsov, B.; Melnikov, A.; Zharov, V.; Khlebtsov, N. Nanotechnology 2006, 17, $1437-1445$.

(38) Nordlander, P.; and C. Oubre,; Prodan, E.; Li, K.; and M. I. Stockman, Nano Lett. 2004, 4, 899-903.

(39) Romero, I.; Aizpurua, J.; Bryant, G. W.; García De Abajo, F. J. Opt. Express 2006, $14,9988$.

(40) Duval, E. Phys. Rev. B 1992, 46, 5795-5797.

(41) Lamb, H. Proc. London Math. Soc. 1882, 13, 189.

(42) Guillet, Y.; Audoin, B.; Ferrié, M.; Ravaine, S. Phys. Rev. B 2012, 86, 35456.

(43) Saviot, L.; Murray, D. B. Phys. Rev. B 2010, 81, 235432.

(44) Neighbours, J. R.; Alers, G. A. Phys. Rev. 1958, 111, 707-712.

(45) Jais, P. M.; Murray, D. B.; Merlin, R.; Bragas, A. V. Nano Lett. 2011, 3685-3689.

(46) Tchebotareva, A. L.; Van Dijk, M. A.; Ruijgrok, P. V.; Fokkema, V.; Hesselberth, M. H. S. Chem. Phys. Chem. 2009, 111-114. 
(47) Stafford, C. M.; Vogt, B. D.; Harrison, C.; April, R. V.; Re, V.; Recei, M.; June, V. 2006, 5095-5099.

(48) Wood, C. D.; Chen, L.; Burkhart, C.; Putz, K. W.; Torkelson, J. M.; Brinson, L. C. Polymer (Guildf). 2015, 75, 161-167.

(49) Chung, P. C.; Green, P. F. Macromolecules 2015, 48, 3991-3996.

(50) Cheng, X.; Putz, K. W.; Wood, C. D.; Brinson, L. C. Macromol. Rapid Commun. 2015, 36, 391-397. 
Graphical TOC Entry

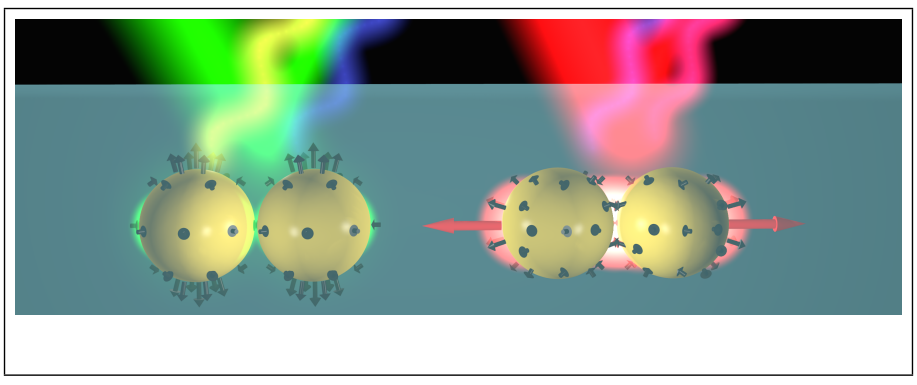

